\title{
Critical aneurysmal dilatation of congenital kinking of the aorta
}

\author{
Osamu Sakai, MD, Masaaki Yamagishi, MD, Keisuke Shuntoh, MD, Takahisa Okano, MD, Yoshiaki Yamada, MD, \\ Katuji Fujiwara, MD, and Nobuo Kitamura, MD, Kyoto, Japan
}

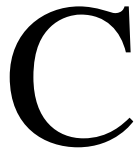
ongenital kinking of the aortic arch is an uncommon anomaly consisting of elongation of the aortic arch with kinking at the level of the ductal ligament. We report a rare case of congenital kinking of the aortic arch with rapid aneurysmal dilatation.

\section{Clinical Summary}

A 13-year-old boy was referred to our hospital for headache and the presence of a cervical bruit. He underwent patch closure of a ventricular septal defect when he was 1 year old. Although the aortic arch anomaly had already been detected at the previous operation, aortic arch reconstruction was not performed because of the lack of clinical symptoms. On admission, systolic blood pressure was $160 \mathrm{~mm} \mathrm{Hg}$ in the right arm and $120 \mathrm{~mm} \mathrm{Hg}$ in the left arm. There was no heart murmur, but a bruit was audible on the left cervical area. The chest radiograph showed a widened left superior mediastinum. The size of the aortic arch had not changed, as evidenced by periodic examination throughout the 12-year period; however, rapid expansion of the aorta was detected on the latest aortogram. There were no collateral vessels originating from the aorta. Three-dimensional (3D) images obtained by means of heli-

From the Department of Pediatric Cardiovascular Surgery, Children's Research Hospital, Kyoto Prefectural University of Medicine, Kyoto, Japan.

Received for publication Feb 13, 2001; accepted for publication Feb 27, 2001.

Address for reprints: Osamu Sakai, MD, Department of Pediatric Cardiovascular Surgery, Children's Research Hospital, Kyoto Prefectural University of Medicine, Kawaramachi, Hirokoji, Kamigyo-ku, Kyoto, 6028566 Japan (E-mail: osakai@koto.kpu-m.ac.jp).

J Thorac Cardiovasc Surg 2001;122:832-3

Copyright $@ 2001$ by The American Association for Thoracic Surgery

$0022-5223 / 2001 \$ 35.00+0 \quad \mathbf{1 2 / 5 4 / 1 1 5 4 1 4}$

doi:10.1067/mtc. 2001.115414 cal computed tomography (CT) showed an elongated serpentine aortic arch with local aneurysmal dilatation and minor dilatation of the proximal left subclavian artery (Figure 1). The largest diameter of the dilated aortic arch was $27.5 \mathrm{~mm}$. Because the latest examinations suggested impending rupture of the aneurysm, an early surgical intervention was considered.

An operation was performed through a repeated median sternotomy. Arterial cannulas were inserted into the ascending aorta and the right femoral artery. Both venae cavae were also cannulated. The right upper part of the body and lower half of the body were continuously perfused throughout the repair process. After institution of moderate hypothermic cardiopulmonary bypass, the aortic arch and descending aorta were carefully dissected. Accurate morphologic orientation of the aortic arch was verified by reference to the 3D helical CT images. The distal aortic arch was elongated and dilated aneurysmally. Its wall was very thin but rigid. The ductal ligament was divided. The aortic arch was clamped just proximal to the origin of the left subclavian artery. The left subclavian artery and the descending aorta were also clamped. The serpentine and dilated vessels were totally resected. The neoaortic arch and the left subclavian artery were reconstructed with a 20-mm Dacron graft with a composite 8-mm graft (Hemashield; Meadox Medicals, Oakland, NJ). All procedures were completed with the heart beating. The resected aorta was $27.5 \mathrm{~cm}$ long, with a lumen that mingled with the aneurysmal dilatation and stenotic narrowing. Microscopic examination of the excised segment demonstrated an irregularly thin and thick wall with disruption of elastic fibers. These findings strongly suggested impending rupture of the dilated aorta. The postoperative course was uneventful. Blood pressure reverted to normal and was equal in both upper extremities.

\section{Discussion}

Congenital kinking of the aortic arch is also termed pseudocoarctation because of certain roentgenographic similarities to simple coarctation of the aorta. However, stenosis of aortic lumen, 


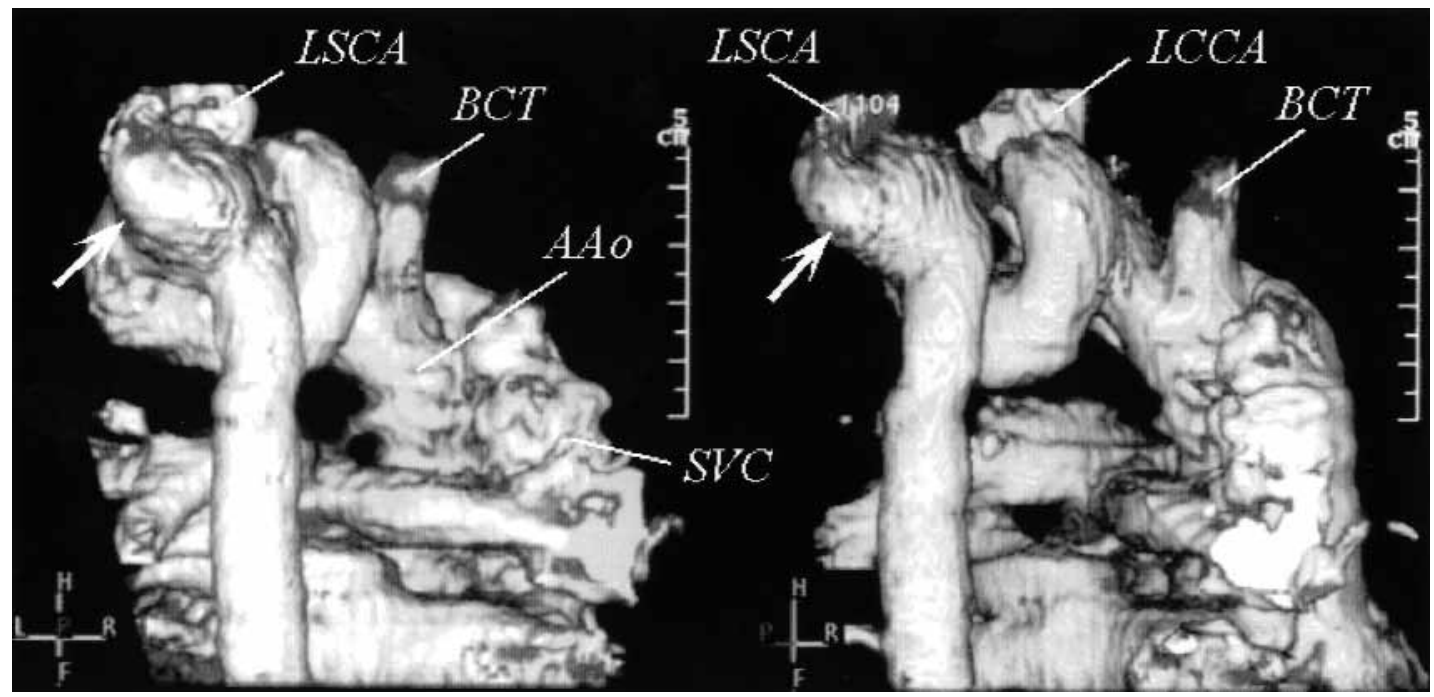

Figure 1. Three-dimensional helical CT demonstrated an elongated serpentine aortic arch with local aneurysmal dilatation: left, posterior view; right, right posterior view; white arrow, aneurysmal dilatation. AAo, Ascending aorta; $B C T$, brachiocephalic trunk; $L C C A$, left common carotid artery; $L S C A$, left subclavian artery; SVC, superior vena cava.

increased pressure gradient across the lesion, and collateral circulation ${ }^{1}$ are not features of kinking of the aortic arch but rather characteristic findings of simple aortic coarctation. The embryopathologic background of congenital kinking of the aortic arch is deficiency of compression of the third through tenth segments of the dorsal aortic roots and the left fourth arch segment. ${ }^{2}$ Morphologic peculiarities consisted of elongation of the aortic arch, which extends high into the superior mediastinum and often above the clavicle. Arch elongation is frequently associated with increased distance between the origins of the left common carotid and left subclavian arteries ${ }^{3}$ and causes kinking or buckling of the aortic isthmus at the attachment of the ductal ligament.

The pathogenesis of aneurysmal dilatation of the kinking aortic arch is presumably related to intraluminal turbulent flow, ${ }^{4}$ and therefore it does not necessarily follow that graft replacement of the aortic arch is required. The precise incidence of aneurysmal dilatation and rupture of kinking of the aorta has not been carefully determined. In the lack of risk of rupture, surgical intervention may not be necessary. However, as demonstrated in our case, surgical intervention should be performed in patients with rapid dilatation of the aortic aneurysm. Turner and associates ${ }^{5}$ also reported aneurysmal dilatation of kinking of the aorta 12 years after the first diagnosis. Hence, once the diagnosis of congenital kinking of the aorta has been established, a periodic follow-up is necessary to identify aneurysmal dilatation.

Compared with conventional aortography, 3D helical CT is less invasive and the most useful technique available for accurate evaluation of the morphologic orientation of the elongated aortic arch.

\section{References}

1. Tsai LM, Fu M, Chang CH, Hung JS. Figure-eight kinking of the aorta (pseudocoarctation) coexistent with coarctation. Chest. 1990;97:1239-40.

2. Hoeffel JC, Henry M, Mentre B, Louis JP, Pernot C. Pseudocoarctation or congenital kinking of the aorta: radiologic considerations. Am Heart J. 1975;89:428-36.

3. Steinberg I, Engle MA, Holswade GR, Hagstrom JWC. Pseudocoarctation of the aorta associated with congenital heart disease: report of ten cases. Am J Roentgenol Radium Ther Nucl Med. 1969;106:1-20.

4. Bahabozorgui S, Bernstein RG, Frater RWM. Pseudocoarctation of the aorta associated with aneurysm formation. Chest. 1972;60:616-7.

5. Turner AF, Swenson BE, Jacobson G, Kay FH. Kinking or buckling of the aorta: case report with complication of aneurysm formation. Am J Roentgenol Radium Ther Nucl Med. 1966;97:411-5. 